Regular article

\title{
Hydroxycinnamic acids and curcumin production in engineered Escherichia coli using heat shock promoters
}

\author{
Joana L. Rodrigues ${ }^{\mathrm{a}, \mathrm{c}, \mathrm{d}, *}$, Márcia R. Couto ${ }^{\mathrm{a}}$, Rafael G. Araújo ${ }^{\mathrm{a}, 1}$, Kristala L.J. Prather ${ }^{\mathrm{b}, \mathrm{c}, \mathrm{d}}$, \\ Leon Kluskens ${ }^{\mathrm{a}, 2}$, Lígia R. Rodrigues ${ }^{\mathrm{a}, \mathrm{c}, \mathrm{d}, *}$ \\ a Centre of Biological Engineering, University of Minho, Campus de Gualtar, 4710-057 Braga, Portugal \\ ${ }^{\mathrm{b}}$ Department of Chemical Engineering, Synthetic Biology Engineering Research Center (SynBERC) Massachusetts Institute of Technology, Cambridge, MA \\ 02139, USA \\ ${ }^{\mathrm{c}}$ MIT-Portugal Program, Cambridge, MA, USA \\ ${ }^{\mathrm{d}}$ MIT-Portugal Program, Lisbon, Portugal
}

\section{A R T I C L E I N F O}

\section{Article history:}

Received 23 October 2016

Received in revised form 21 March 2017

Accepted 20 May 2017

Available online 22 May 2017

\section{Keywords:}

Heat shock promoters

E. coli

Biosynthetic pathway

Caffeic acid

p-Coumaric acid

Curcumin

\begin{abstract}
A B S T R A C T
Hydroxycinnamic acids and curcumin are compounds with great therapeutic potential, including anticancer properties. In this study, p-coumaric acid, caffeic acid and curcumin were produced in Escherichia coli. Their production was induced by heat using the $d n a K$ and $i b p A$ heat shock promoters. The ribosome binding site (RBS) used was tested and further optimized for each gene to assure an efficient translation. p-Coumaric acid was successfully produced from tyrosine and caffeic acid was produced either from tyrosine or $p$-coumaric acid using tyrosine ammonia lyase (TAL) from Rhodotorula glutinis, 4-coumarate 3-hydroxylase $(\mathrm{C} 3 \mathrm{H})$ from Saccharothrix espanaensis or cytochrome P450 CYP199A2 from Rhodopseudomonas palustris. The highest $p$-coumaric acid production obtained was $2.5 \mathrm{mM}$; caffeic acid production reached $370 \mu \mathrm{M}$. Regarding curcumin, $17 \mu \mathrm{M}$ was produced using 4-coumarate-CoA ligase (4CL1) from Arabidopsis thaliana, diketide-CoA synthase (DCS) and curcumin synthase 1 (CURS1) from Curcuma longa. Stronger RBSs and/or different induction conditions should be further evaluated to optimize those production levels. Herein it was demonstrated that the biosynthetic pathway of $p$-coumaric acid, caffeic acid and curcumin in $E$. coli can be triggered by using heat shock promoters, suggesting its potential for the development of new industrial bioprocesses or even new bacterial therapies.
\end{abstract}

(c) 2017 Elsevier B.V. All rights reserved.

\section{Introduction}

Hydroxycinnamic acids such as p-coumaric and caffeic acids are phenylpropanoids naturally synthesized by plants from amino acids. They can be found in several fruits and vegetables, and have a well-known antioxidant activity. In addition, they have been

Abbreviations: 4CL, 4-coumarate-CoA ligase; $\mathrm{C} 3 \mathrm{H}, 4$-coumarate 3-hydroxylase; CURS1, curcumin synthase 1 ; DCS, diketide-CoA synthase; GFP, green fluorescence protein; IPTG, isopropyl $\beta$-D-1-thiogalactopyranoside; OD, optical density; RBS, ribosome binding site; TAL, tyrosine ammonia lyase; TIR, translation initiation rate; UHPLC, ultra-high-performance liquid chromatography.

* Corresponding authors at: Centre of Biological Engineering, University of Minho, Campus de Gualtar, 4710-057 Braga, Portugal.

E-mail addresses: joana.joanalucia@deb.uminho.pt

(J.L. Rodrigues), marciacouto93@gmail.com (M.R. Couto), rafa.gomes.ar@gmail.com (R.G. Araújo), kljp@mit.edu (K.L.J. Prather), lrmr@deb.uminho.pt (L.R. Rodrigues).

1 Biorefinery Group, Food Research Department, School of Chemistry, Autonomous University of Coahuila, 25280 Saltillo, Coahuila, Mexico.

2 Deceased on April 1st 2016. reported to have other benefits including anti-inflammatory and anticancer activities [1,2]. Due to its natural radical scavenging properties, studies showed that $p$-coumaric acid protects the rat heart against the oxidative stress caused by the anticancer drug doxorubicin and that the pre-treatment and co-administration of $p$-coumaric acid can be highly beneficial $[2,3]$. In addition to several reports showing its anticancer and anti-apoptosis properties [1], caffeic acid has also been shown to possess antiviral and antidiabetic activities [4-6]. All of these beneficial properties encourage the application of $p$-coumaric and caffeic acids in pharmaceuticals and healthy or functional foods. In addition, these hydroxycinnamic acids are precursors to an enormous array of secondary metabolites with desirable and relevant properties, such as curcumin. Curcumin is a polyphenolic compound that has been used as a food additive, as well as in traditional medicine [7] due to its several therapeutic properties including anticancer, antioxidant, anti-inflammatory, anti-HIV, anti-Alzeimer's and anti-Parkinson [8-11]. All of these compounds are accumulated at low levels in plants and their extraction is complex, low, inefficient, environ- 
(A)<smiles>O=C(O)/C=C/c1ccc(O)c(O)c1</smiles>

(B)<smiles>COc1cc(/C=C/C(=O)O)ccc1O</smiles><smiles>COc1cc(/C=C/C(=O)CC(=O)OCc2ccccc2)ccc1O</smiles>

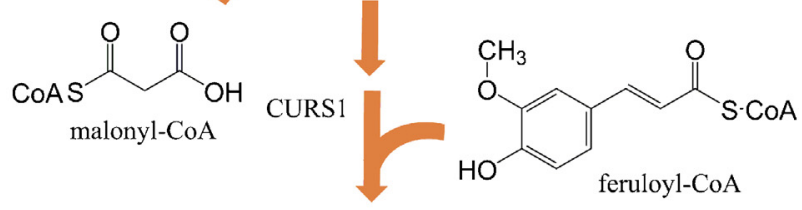<smiles>COc1cc(/C=C/C(=O)/C=C(O)/C=C/c2ccc(O)c(OC)c2)ccc1O</smiles>

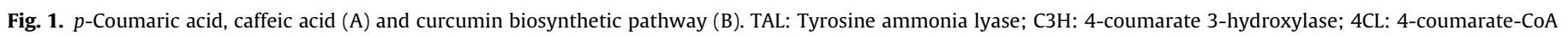
ligase; DCS: diketide-CoA synthase; CURS1: curcumin synthase 1 .

mentally unfriendly and expensive [12]. In addition, their isolation as pure compounds remains inefficient and their availability is limited by regional variations and seasonality [13]. Also, their chemical synthesis is very difficult. Therefore, microbial conversion comprises a promising alternative for the production of hydroxycinnamic acids and curcumin. p-Coumaric acid, caffeic acid and curcumin were recently produced in Escherichia coli using different artificial biosynthetic pathways [14-19]. Previously, we reported the production of hydroxycinnamic acids and curcumin in E. coli using the biosynthetic pathway illustrated in Fig. $1[17,18]$.

$E$. coli has been the host of choice for the expression of recombinant proteins given its ability to produce high quantities at low costs. However, in large-scale productions, chemical inducers, such as isopropyl $\beta$-D-1-thiogalactopyranoside (IPTG), can be expensive and toxic $[20,21]$ and their presence in waste effluents or in the final product must be eliminated, especially in the production of pharmaceutical-grade proteins and other products intended for human use [22]. Constitutive promoters or promoters induced by starvation of an essential nutrient, or by shift in a physical or physicochemical factor, such as temperature or $\mathrm{pH}$, allow an inducer-free environment for heterologous protein expression [23]. Thermal induction in $E$. coli is carried out by increasing the temperature (usually $37^{\circ} \mathrm{C}$ ) to $42^{\circ} \mathrm{C}$ or higher for a certain period and then, shifting it down [24-31]. A thermal induction strategy has the potential of reducing the fermentation cost since expensive chemicals or special media are not required. In addition, it simplifies the downstream processing since culture handling and contamination risks are minimized [24,32]. All of these aspects are very important when producing therapeutic recombinant proteins and products at an industrial scale [24]. Induction by heat can also be very advantageous in therapeutic approaches, for example in bacterial therapies. These therapies can be combined with laser or ultrasound treatments and the temperature increase would trigger the production in situ of the desired compounds, such as hydroxycinnamic acids and curcumin.

Due to the need of finding and characterizing new parts to use in synthetic biology approaches and the advantages of using promoters not chemically induced, we previously studied $E$. coli heat shock promoters [33]. The $d n a K$ and $i b p A$ heat shock promoters were used with a synthetic ribosome binding site (RBS) and the green fluorescence protein (GFP) to design and construct stress probes, which were further used to evaluate the promoter strength and their potential use in synthetic biology applications [33]. In the current study, the heat shock induction system was coupled to the artificial biosynthetic pathways leading to the production of $p$-coumaric acid, caffeic acid and curcumin. The dnaK and $i b p A$ promoters and different synthetic RBSs with several strengths were used. The results gathered herein demonstrate that $p$-coumaric acid, caffeic acid and curcumin can be produced in E. coli using heat shock promoters and that synthetic biology tools can help to improve their production.

\section{Materials and methods}

\subsection{Bacterial strains, plasmids and chemicals}

E. coli NZY5 $\alpha$ competent cells (NZYTech, Lisbon, Portugal) were used for molecular cloning and vector propagation and E. coli K12 MG1655(DE3) [34] was used as host. E. coli K-12 ER2925 (NEB, Ipswich, MA, USA) competent cells were used whenever restriction endonucleases sensitive to E. coli K-12 methylation patterns were required. Table 1 summarizes the characteristics of all strains and plasmids used. Synthesis and amplification of TAL, C3H, CYP199A2, Pdr, Pux, 4CL1, DCS and CURS1 was previously described $[17,18]$. The DNA sequences of the codon-optimized genes are provided in Table S1. pAC-4CL1 plasmid was kindly provided by Claudia Schmidt-Dannert [35] (Addgene plasmid \# 35947).

L-Tyrosine, $p$-coumaric and caffeic acid were purchased from Sigma-Aldrich (Steinheim, Germany); ferulic acid from Acros (Geel, Belgium); curcumin from Fisher Scientific (Loughborough, UK) and Luria-Bertani (LB) medium from NZYTech (Lisbon, Portugal). Glucose (Acros), $\mathrm{Na}_{2} \mathrm{HPO}_{4}$ (Scharlau, Sentmenat, Spain), $\mathrm{MgSO}_{4}, \mathrm{KH}_{2} \mathrm{PO}_{4}$ (Riel-deHaën, Seelze, Germany), $\mathrm{NH}_{4} \mathrm{Cl}, \mathrm{NaCl}$, $\mathrm{CaCO}_{3}$ (Panreac, Barcelona, Spain) and thiamine (Fisher Scientific, Loughborough, UK) were used to prepare the M9 modified salt medium. The following mineral traces and vitamins were 
Table 1

Bacterial strains and plasmids used in this study.

\begin{tabular}{|c|c|c|}
\hline Strains & Relevant Genotype & Source \\
\hline E. coli $\mathrm{NZY} 5 \alpha$ & $\begin{array}{l}\text { fhuA2 } \Delta(\operatorname{argF-lacZ)U169} \text { phoA glnV44 } \Phi 80 \Delta(\text { lacZ)M15 gyrA96 recA1 relA1 } \\
\text { endA1 thi-1 hsdR17 }\end{array}$ & NZYTech \\
\hline E. coli K-12 ER2925 & $\begin{array}{l}\text { ara-14 leuB6 fhuA31 lacY1 tsx78 glnV44 galK2 galT22 mcrA dcm-6 hisG4 rfbD1 } \\
\text { R(zgb210:Tn10)TetS endA1 rpsL136 dam13:Tn9 xylA-5 mtl-1 thi-1 mcrB1 hsdR2 }\end{array}$ & NEB (E4109) \\
\hline E. coli K-12 MG1655(DE3) & $F^{-} \lambda^{-} i l v G^{-} r f b^{-} 50 r p h^{-} 1 \lambda(\mathrm{DE} 3)$ & [34] \\
\hline Plasmids & Construct & Source \\
\hline pETDuet-1 & ColE1(pBR322) ori, lacI, double T7lac, Amp ${ }^{\mathrm{R}}$ & Novagen \\
\hline pCDFDuet-1 & CloDF13 ori, lacI, double T7lac, Strep ${ }^{R}$ & \\
\hline pRSFDuet-1 & RSF ori, lacI, double T7lac, $\mathrm{Kan}^{\mathrm{R}}$ & \\
\hline pUC57_TAL & pUC57 carrying codon-optimized TAL from Rhodotorula glutinis & GenScript \\
\hline pUC57_C3H & pUC57 carrying codon-optimized C3H from Saccharothrix espanaensis & \\
\hline pUC57_CYP199A2 & pUC57 carrying codon-optimized CYP199A2 from Rhodopseudomonas palustris & \\
\hline pUC57_DCS & pUC57 carrying codon-optimized DCS from Curcuma longa & NZYTech \\
\hline pUC57_CURS1 & pUC57 carrying codon-optimized CURS1 from C. longa & \\
\hline $\mathrm{pAC}-4 \mathrm{CL} 1$ & P15A ori, $\mathrm{P}_{\text {lac }}, \mathrm{Cm}^{\mathrm{R}}$, pACYC184-derived plasmid carrying 4CL1 from Arabidopsis thaliana & Addgene (\#35947) \\
\hline pCDF_TAL & pCDFDuet- 1 carrying TAL & [18] \\
\hline pCDF_CYP & pCDFDuet-1 carrying CYP199A2 & \\
\hline pET_Pdr_Pux_op & pETDuet-1 carrying Pdr from Pseudomonas putida and Pux from $R$. palustris in an operon & \\
\hline pCDF_dnaKp_RBS1_TAL & pCDFDuet_TAL carrying dnaK promoter and RBS1 & This study \\
\hline pRSF_dnaKp_RBS1_TAL & pRSFDuet- 1 carrying TAL, dnaK promoter and RBS1 & This study \\
\hline pCDF_dnaKp_RBS1_CYP & pCDFDuet_CYP carrying dnaK promoter and RBS1 & This study \\
\hline pCDF_dnaKp_RBS1_C3H & pCDFDuet- 1 carrying $\mathrm{C} 3 \mathrm{H}$, dnaK promoter and RBS1 & This study \\
\hline pRSF_dnaKp_RBS1_C3H & pRSFDuet-1 carrying $\mathrm{C} 3 \mathrm{H}$, dnaK promoter and RBS1 & This study \\
\hline pET_dnaKp_RBS1_Pdr_Pux_op & pETDuet- 1 carrying Pdr and Pux in an operon, dnaK promoter and RBS1 & This study \\
\hline pCDF_dnaKp_RBS2_TAL & pCDFDuet- 1 carrying TAL, dnaK promoter and RBS2 & This study \\
\hline pET_dnaKp_RBS2_TAL & pETDuet-1 carrying TAL, dnaK promoter and RBS2 & This study \\
\hline pET_ibpAp_RBS2_C3H & pETDuet- 1 carrying $\mathrm{C} 3 \mathrm{H}, i b p A$ promoter and $\mathrm{RBS} 2$ & This study \\
\hline pET_dnaKp_RBS2_TAL_ibpAp_RBS2_C3H & pET_dnaKp_RBS2_TAL carrying $\mathrm{C} 3 \mathrm{H}, i b p A$ promoter and $\mathrm{RBS} 2$ & This study \\
\hline pCDF_dnaKp_RBS2_CYP & pCDFDuet-1 carrying CYP199A2, dnaK promoter and RBS2 & This study \\
\hline pET_ibpAp_RBS2_Pdr_Pux_op & pETDuet- 1 carrying Pdr and Pux in an operon, ibpA promoter and RBS2 & This study \\
\hline pRSF_dnaKp_RBS1_CURS1 & pRSFDuet- 1 carrying CURS1, dnaK promoter and RBS1 & This study \\
\hline pCDF_dnaKp_RBS1_DCS & pCDFDuet-1 carrying DCS, dnaK promoter and RBS1 & This study \\
\hline pRSF_dnaKp_RBS2_CURS1 & pRSFDuet- 1 carrying CURS1, dnaK promoter and RBS2 & This study \\
\hline pCDF_dnaKp_RBS2_DCS & pCDFDuet-1 carrying DCS, dnaK promoter and RBS2 & This study \\
\hline
\end{tabular}

supplemented to M9 Medium: $\mathrm{FeCl}_{3}, \mathrm{ZnCl}_{2}, \mathrm{CoCl}_{2}, \mathrm{CuCl}_{2}$, nicotinic acid (Riedel-deHaën), $\mathrm{NaMoO}_{4}, \mathrm{H}_{2} \mathrm{BO}_{3}$, pyridoxine, biotin, folic acid (Merck), riboflavin and pantothenic acid (Sigma Aldrich). Ampicillin (Applichem, Darmstadt, Germany), chloramphenicol, kanamycin (NZYTech) and spectinomycin (Panreac) were used when necessary.

\subsection{Design of the dnaK promoter and RBS}

The dnaK and $i b p A$ heat shock promoter sequences used were obtained from the NCBI (National Center for Biotechnology Information) database and previously described by Rodrigues, Sousa, Prather, Kluskens and Rodrigues [33]. These promoters and the synthetic RBS were used to replace the T7 promoter and the RBSs of the plasmids from Novagen (Table 1). The RBSs were designed using the software RBS Calculator v1.1 [36]. RBS1 was previously designed by Rodrigues, Sousa, Prather, Kluskens and Rodrigues [33] and the sequence used herein was the same for all genes. The other synthetic RBSs (RBS2) (Table S2) were designed to improve the expression of each gene and vary in the sequence, spacer length and translation initiation rate (TIR). The TIR for each gene was set to the "maximum" value.

\subsection{Construction of the plasmids}

The dnaK promoter with synthetic RBS1 was cloned in the plasmids pCDF_TAL and pCDF_CYP previously constructed [18]. For the other constructions the heat shock promoters and the synthetic RBS were first cloned in an empty plasmid, and only afterwards the genes TAL, C3H, Pdr_Pux_operon (GenBank accession numbers CAE27313.1 and BAN13287.1), CURS1 and DCS were cloned. This approach was adopted to avoid digestion of the genes by the restriction enzymes required to clone the promoter. A restriction site in the FW primer was used to assist with colony screening. The primers (Invitrogen/Life Technologies, Carlsbad, CA, USA) used are summarized in Table S3.

Plasmid DNA was isolated using NucleoSpin ${ }^{\circledR}$ Plasmid Miniprep Kit (Macherey-Nagel, Düren, Germany). The genes were amplified by PCR using KAPA HiFi DNA Polymerase (Kapa Biosystems, Wilmington, MA, USA). DNA fragments were purified from agarose using Gel DNA Recovery Kit (Zymo Research, Orange, CA, USA). Plasmid DNA and genes were quantified in a NanoDrop instrument (ND-1000, Thermo Scientific, Wilmington, DE, USA) and were digested with the appropriate restriction endonucleases (Tables S2 and S3) (NEB) for $3 \mathrm{~h}$ and purified using DNA Clean and Concentrator Kit (Zymo Research). Ligation was performed at room temperature for $1 \mathrm{~h}$ with T4 DNA ligase (NEB). Chemical transformation (heat shock method) was carried out using E. coli NZY5 $\alpha$ or E. coli K-12 ER2925 competent cells. All constructed plasmids described herein (Table 1) were verified by colony PCR and/or digestion and confirmed by sequencing (GATC Biotech, Konstanz, Germany). After confirmation, E. coli K-12 MG1655(DE3) was transformed with the plasmids. All the kits and enzymes were used according to the instructions provided by the manufacturers.

\section{4. p-Coumaric acid, caffeic acid and curcumin production}

E. coli cells for gene cloning, plasmid propagation, and inoculum preparation were grown in LB medium at $37^{\circ} \mathrm{C}$ and under shaking conditions (200 rpm).

For $p$-coumaric acid, caffeic acid and curcumin production, cultures were grown at $37^{\circ} \mathrm{C}$ in $50 \mathrm{~mL}$ LB up to an optical density at 
$600 \mathrm{~nm}\left(\mathrm{OD}_{600}\right)$ of $0.3-0.4$. After that, a heat shock at $48^{\circ} \mathrm{C}$ for $5 \mathrm{~min}$ was performed in a shaking water bath to induce gene expression. Next, the culture was incubated for $5 \mathrm{~h}$ at 26 or $37^{\circ} \mathrm{C}$. Control flasks were kept at $37^{\circ} \mathrm{C}$.

The cells were harvested by centrifugation, suspended, and incubated at 26 or $37^{\circ} \mathrm{C}$ for $63 \mathrm{~h}$ in $50 \mathrm{~mL}$ modified M9 minimal salt medium containing (per liter): glucose $(40 \mathrm{~g}), \mathrm{Na}_{2} \mathrm{HPO}_{4}$ (6 g), $\mathrm{KH}_{2} \mathrm{PO}_{4}$ (3 g), $\mathrm{NH}_{4} \mathrm{Cl}$ (1 g), $\mathrm{NaCl}$ (0.5 g), $\mathrm{CaCl}_{2}$ (15 mg), $\mathrm{MgSO}_{4}$ $(110 \mathrm{mg})$, thiamine $(340 \mathrm{mg})$ and $\mathrm{CaCO}_{3}(5 \mathrm{~g})$ (to control the $\mathrm{pH}$ ). Trace elements [ $\mathrm{FeCl}_{3}$ (54 mg), $\mathrm{ZnCl}_{2}$ ( $4 \mathrm{mg}$ ), $\mathrm{CoCl}_{2}(4 \mathrm{mg}), \mathrm{NaMoO}_{4}$ (4 mg), $\mathrm{CuCl}_{2}(2 \mathrm{mg})$ and $\mathrm{H}_{2} \mathrm{BO}_{3}(1 \mathrm{mg})$ ] and vitamins [riboflavin $(0.84 \mathrm{mg})$, folic acid $(0.084 \mathrm{mg})$, nicotinic acid $(12.2 \mathrm{mg})$, pyridoxine $(2.8 \mathrm{mg})$, biotin $(0.12 \mathrm{mg})$ and pantothenic acid $(10.8 \mathrm{mg})]$ were supplemented to the medium. Depending on the plas$\operatorname{mid}(\mathrm{s})$ present in the strain, $100 \mu \mathrm{g} / \mathrm{mL}$ of ampicillin, $100 \mu \mathrm{g} / \mathrm{mL}$ of spectinomycin, $30 \mu \mathrm{g} / \mathrm{mL}$ of chloramphenicol and $/$ or $50 \mu \mathrm{g} / \mathrm{mL}$ of kanamycin were added. Shake flasks were incubated for $30 \mathrm{~min}$ at $26^{\circ} \mathrm{C}$ or $37^{\circ} \mathrm{C}$ to stabilize the initial temperature and then they were immersed in a shaking water bath at $48^{\circ} \mathrm{C}$. After 5 min under this temperature, the shake flasks were placed back in the incubator at $26^{\circ} \mathrm{C}$ or $37^{\circ} \mathrm{C}$. Control flasks were kept at $26^{\circ} \mathrm{C}$ or $37^{\circ} \mathrm{C}$ throughout the experiment. Substrates were added at time 0 of induction in M9 medium: tyrosine ( $3 \mathrm{mM}), p$-coumaric acid ( $2 \mathrm{mM}$ ) and ferulic acid $(2 \mathrm{mM})$. Supernatant samples $(1.5 \mathrm{~mL})$ were collected for the analysis of $p$-coumaric and caffeic acids, while for curcumin $2 \mathrm{~mL}$ of culture broth with cells (whole broth) were collected. All the experiments were conducted in triplicate.

\subsection{Curcumin extraction}

For subsequent curcumin analysis, $2 \mathrm{~mL}$ of whole broth was adjusted to $\mathrm{pH} 3.0$ with $6 \mathrm{M} \mathrm{HCl}$. Then, curcumin was extracted with an equal volume of ethyl acetate. The extracts were concentrated by solvent evaporation in a fume hood, suspended with $200 \mu \mathrm{L}$ of acetonitrile and subjected to product analysis by ultrahigh-performance liquid chromatography (UHPLC).

\subsection{UHPLC analysis of products}

UHPLC analysis was used to quantify $p$-coumaric acid, caffeic acid, ferulic acid and curcumin using the Shimadzu Nexera-X2 (Shimadzu Corporation, Kyoto, Japan) (CBM-20A system controller, LC-30AD pump unit, DGU-20A 5R degasser unit, SPD-M20A detector unit, SIL-30AC autosampler unit, CTO-20AC column oven) system and a Grace Alltech Platinum EPS C18 column $(3 \mu \mathrm{m}$, $150 \mathrm{~mm} \times 4.6 \mathrm{~mm}$ ) (Grace, Columbia, MD, USA). Mobile phases A and $B$ were composed of water $(0.1 \%$ trifluoroacetic acid) and acetonitrile, respectively. For hydroxycinnamic acid quantification the following gradient was used at a flow rate of $1 \mathrm{~mL} / \mathrm{min}$ : $10-20 \%$ acetonitrile (mobile phase B) for 16 min. Quantification was based on the peak areas at $310 \mathrm{~nm}$ for $p$-coumaric acid, caffeic acid and ferulic acid. The retention times of caffeic acid, $p$-coumaric acid and ferulic acid were $6.9,8.9$ and $9.7 \mathrm{~min}$, respectively. For curcumin quantification, a gradient of $40-43 \%$ acetonitrile (mobile phase B) for $15 \mathrm{~min}$ and $43 \%$ acetonitrile for an additional $5 \mathrm{~min}$ was used. Curcumin was detected at $425 \mathrm{~nm}$ of absorbance and the retention time $17.6 \mathrm{~min}$.

\subsection{Protein analysis}

E. coli K-12 MG1655 (DE3) cells harboring pCDFDuet-1, pCDF_dnaKp_RBS1_TAL and pCDF_dnaKp_RBS2_TAL were grown in $\mathrm{LB}$ at $37^{\circ} \mathrm{C}$ to an $\mathrm{OD}_{600}$ of $0.3-0.4$. Samples were centrifuged and the cells were resuspended in phosphate-buffered saline (PBS: $137 \mathrm{mM} \mathrm{NaCl}, 2.7 \mathrm{mM} \mathrm{KCl}, 10 \mathrm{mM} \mathrm{Na}_{2} \mathrm{PO}_{4}, 1.8 \mathrm{mM} \mathrm{KH}_{2} \mathrm{PO}_{4}, \mathrm{pH} 7.4$ ) and were further disrupted by sonication on ice for 3 min. After centrifugation, the protein concentration from the resulting supernatant was determined using the Bradford Reagent (Sigma-Aldrich) with bovine serum albumin (BSA) (NEB) as a standard, according to the manufacturers' instructions. The expression levels of TAL were evaluated through sodium dodecyl sulfate polyacrylamide gel electrophoresis (SDS-PAGE) (4\% stacking gel and 10\% running gel). Samples containing soluble or insoluble protein fraction were mixed with $2 \mathrm{X}$ sample buffer (65.8 mM Tris- $\mathrm{HCl} \mathrm{pH}$ 6.8, 2.1\% SDS, $26.3 \%$ glycerol, $0.01 \%$ bromophenol blue) and $\beta$-mercaptoethanol and were denatured in a heating block at $100^{\circ} \mathrm{C}$ for $5 \mathrm{~min}$. The protein marker used was Spectra ${ }^{\mathrm{TM}}$ Multicolor High Range Protein (Thermo Scientific). After electrophoresis, the gel was stained using Coomassie Blue R-250 for $30 \mathrm{~min}$ and de-stained using distilled water until a clear background was achieved.

\section{Results and discussion}

\section{1. p-Coumaric and caffeic acids production}

p-Coumaric acid was produced using tyrosine ammonia lyase (TAL) from $R$. glutinis that converts tyrosine to $p$-coumaric acid. To convert $p$-coumaric acid to caffeic acid, 4-coumarate 3-hydroxylase (C3H) from S. espanaensis or cytochrome P450 CYP199A2 from $R$. palustris was used. CYP199A2 only converts $p$-coumaric acid efficiently to caffeic acid in the presence of its redox partners, Pux (palustrisredoxin) from R. palustris and Pdr (putidaredoxin reductase) from Pseudomonas putida. The expression of $p$-coumaric acid and caffeic acid biosynthetic genes was triggered by using heat shock promoters and a heat shock of $48^{\circ} \mathrm{C}$ during 5 min was used. The strategy used was based on previous results regarding the production of caffeic acid using plasmids with T7 promoters [18]. The plasmids pCDFDuet- 1 and pRSFDuet- 1 were chosen since they enabled the highest $p$-coumaric acid and caffeic acid production. As expected, the pRSFDuet-1 plasmid carrying the heat shock promoter enabled a significantly higher $p$-coumaric acid $(119.6 \mu \mathrm{M})$ $(p$-value $<0.006)$ and caffeic $(13.7 \mu \mathrm{M})(p$-value $<0.04)$ acid production than the pCDFDuet-1 plasmid (Fig. 2, bars (2) and (4) compared to bars (1) and (3), respectively). When TAL and $\mathrm{C} 3 \mathrm{H}$ were combined different plasmids were tested (Fig. 2, bars (6) and (7)) but the differences observed in the productions obtained were not statistically significant ( $p$-value $>0.09$ for caffeic acid production and $p$-value $>0.25$ for $p$-coumaric acid production). In addition, the productions of $p$-coumaric acid and caffeic acid were comparable or slightly higher than when TAL and $\mathrm{C} 3 \mathrm{H}$ were tested separately (Fig. 2, bars (1)-(4)). This is in accordance with most of the results previously obtained using different combinations of plasmids carrying TAL and $\mathrm{C} 3 \mathrm{H}$ [18]. It was found that tyrosine is converted faster if $p$-coumaric acid is being converted to the next product which results in higher production of $p$-coumaric acid that never limits the production of caffeic acid. In addition, the caffeic acid increase can be related to the lower concentration of $p$-coumaric acid in the medium (compared to when it is added directly as substrate) that reduces its toxic effect to the cells, as previously described in the literature [18,35,37].

Surprisingly, the results herein obtained with CYP199A2 in pCDFDuet-1 and Pdr and Pux redox partners in pETDuet-1 (Fig. 2, bar (5)) were not in accordance with our previous results [18], since Pdr and Pux expression in pETDuet- 1 using heat shock promoters led to a higher caffeic acid concentration than that found in the $\mathrm{C} 3 \mathrm{H}$ strategy (Fig. 2, bars (3) and (4)). The production was significantly higher when compared to pCDF_dnaKp_RBS1_C3H ( $p$-value $<0.02$ ), while in the case of pRSF_dnaKp_RBS1_C3H the differences in the results are not statistically significant ( $p$-value $>0.07$ ). When tyrosine is added as substrate (Fig. 2, bar (8)), the production of $p$-coumaric acid and caffeic acid is also significantly higher when 


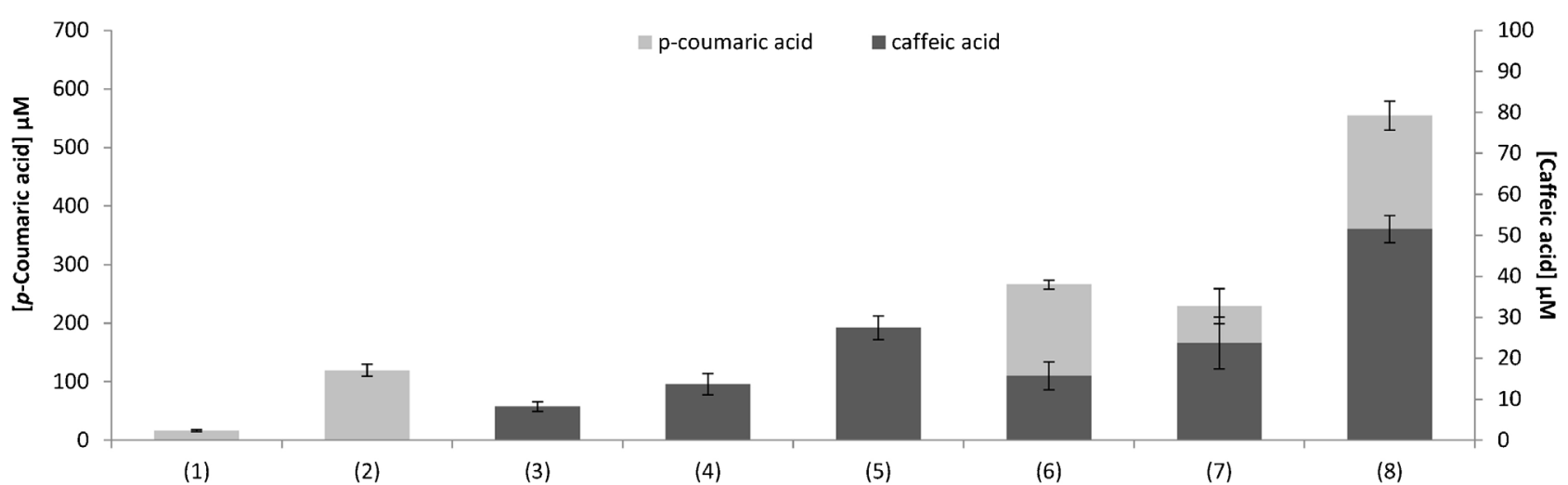

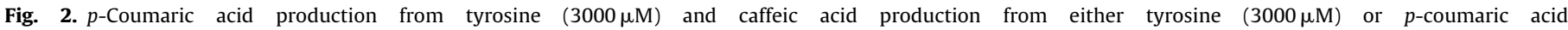

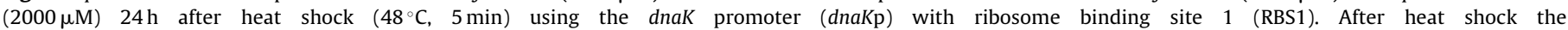

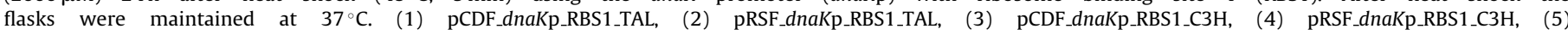

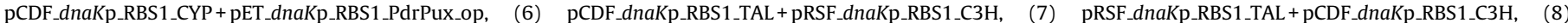

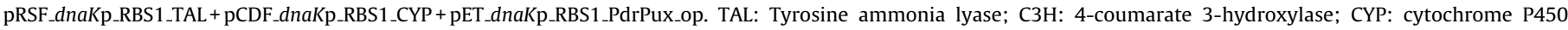
CYP199A2. Error bars are standard deviations from triplicate experiments.

compared to the case where $\mathrm{C} 3 \mathrm{H}$ is used to produce caffeic acid (Fig. 2, bar (7)) ( $p$-values <0.01). This discrepancy between the results obtained for caffeic acid production using the T7 promoter or the $d n a K$ promoter imposed a cautious evaluation of the $d n a K$ promoter constructs and the RBS strength, which are discussed below.

In general, the production levels obtained using a heat shock induction were much lower than the results obtained using IPTG and the T7 promoter [18], which was expected considering that the $\mathrm{T} 7$ promoter is stronger than the dnaK promoter. In our previous study [33], the expression of the dnaK promoter was studied and it was demonstrated that testing and optimizing the RBS is crucial to improve the translational efficiency. In the current study, we first used the $d n a K$ promoter and RBS that previously showed the best performance with GFP expression. A strong RBS with high translation initiation rate (TIR) led to high translation efficiency. This efficiency is completely dependent on the secondary mRNA structure, which is influenced by the sequence upstream of the RBS (promoter sequence), spacer length between the Shine-Dalgarno (SD) sequence and the AUG codon, as well as the beginning of the downstream coding DNA sequence (CDS) $[20,36]$. In addition, to calculate the TIR, the RBS calculator software also simulates the annealing of the ribosome to the RBS and predicts the mRNA secondary structure $[36,38]$. All RBS sequences should be designed to minimize the formation of mRNA secondary structure. All this ensures that the protein expression levels and the productions are optimized in bacteria. Although the TIR of the synthetic RBS was high when GFP expression was considered ( $T I R=175751.22$ ), it was found to be low for TAL (TIR = 2018.90) or C3H (TIR=558.5) expression. This may explain why the $p$-coumaric acid production was higher when compared to the caffeic acid production (Fig. 2, bars (1) and (2), compared to bars (3) and (4), respectively). In addition, TAL expression was found previously to be higher than $\mathrm{C} 3 \mathrm{H}$ using T7 promoters [39], and the highest product yield regarding $p$ coumaric acid was almost two times higher than caffeic acid [18]. Regarding the differences observed in the production whenever using different plasmids, since the TIR is equal in both pCDFDuet- 1 and pRSFDuet-1, but pRSFduet-1 is a higher copy number plasmid, this plasmid gave higher $p$-coumaric acid and caffeic acid productions, as expected.

The results obtained when CYP199A2 and its redox partners were used to produce caffeic acid may also be explained by the TIR (Fig. 2, bar (5)). Although the TIR value of 5372.9 in CYP199A2 expression was not very high as compared to the GFP expression, it was significantly higher when compared to the TAL or C3H expres- sion. The TIR was also relatively high in the Pdr and Pux operon $(T I R=5813.16)$. This could explain why caffeic acid production was higher when using CYP199A2 and Pdr and Pux redox partners in pETDuet-1. When $p$-coumaric acid was not added to the culture medium and TAL was used to produce it (Fig. 2, bar (8)), the $p$-coumaric acid and the caffeic acid production increased significantly probably due to the same reasons stated above relative to $\mathrm{C} 3 \mathrm{H}$.

In order to improve $p$-coumaric acid and caffeic acid production, a new synthetic RBS (RBS2) was designed for each gene using the RBS Calculator software. This new synthetic RBS showed a different sequence, spacer length and TIR for each gene. For instance, in the TAL case, the synthetic RBS had a TIR $=135374.30$ and the TAL gene was cloned in pETDuet-1 and pCDFDuet-1 plasmids (Fig. 3, bars (1) and (2)). The plasmid pETDuet-1 was chosen to construct the curcuminoids biosynthetic pathway previously described [17]. Since this is a very complex pathway we decided to use the same plasmids for each gene of the pathway changing only the promoters (Section 3.2 for more information). The $p$-coumaric acid titers obtained using these plasmids were surprisingly high, especially in comparison to the ones obtained using the T7 promoter and the same plasmids. The production obtained with the medium copy number plasmid pET_dnaKp_RBS2_TAL was 2.8 times higher than pET_TAL that possessed a TIR $=15436.31$ and reached the same level of the high copy number plasmid pRSF_TAL (TIR=27709.67) [18]. The production of $p$-coumaric acid using the low copy number plasmid pCDFDuet_dnaKp_RBS2_TAL was 97.8 times higher than the one obtained with pCDFDuet_dnaKp_RBS1_TAL and 1.4 times higher than pCDF_TAL with T7 promoter $(\mathrm{TIR}=27709.67)$ [18]. These results prove that the TIR value can have a high influence on translation and in the production of the target compounds. Furthermore, the heat shock promoters may enable a higher production of a specific compound than the T7 promoters. The controls with no heat shock (Fig. 3, solid bars) were found to produce more $p$-coumaric acid than the experiments with a 5 min temperature shift to $48^{\circ} \mathrm{C}$ (Fig. 3, slashed bars). The high production values obtained at $37^{\circ} \mathrm{C}$ were not unexpected since the genes regulated by the dnaK promoter were shown to be highly expressed even at this temperature [33]. This was the motivation to use $37^{\circ} \mathrm{C}$ as the control temperature instead of $26^{\circ} \mathrm{C}$, although previously we found that the production with the T7 promoter was higher at $26^{\circ} \mathrm{C}$ [18]. In addition, although the gene expression is significantly higher after heat shock conditions, this heat shock response caused by the stressful condition only lasts a few minutes [33]. Therefore, the increase in genes translation level is only affected 


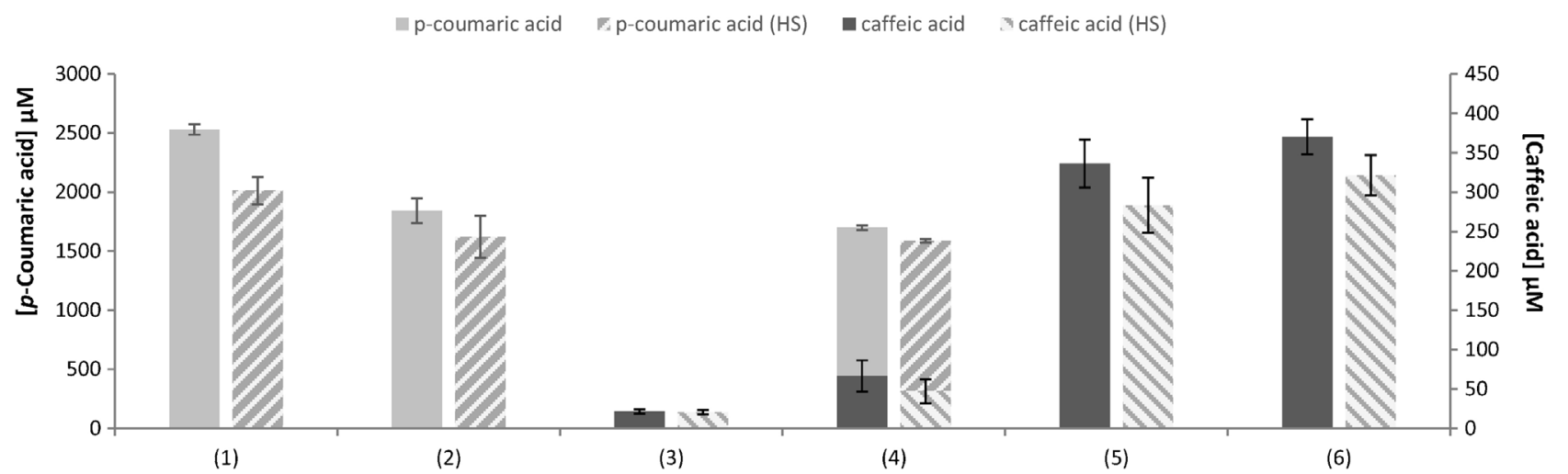

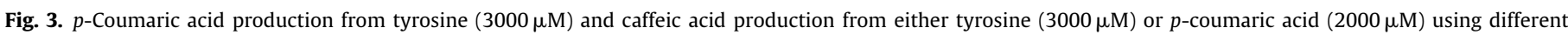

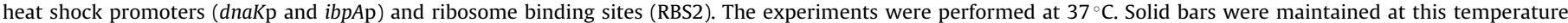

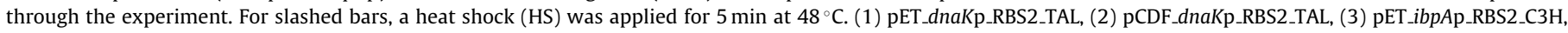

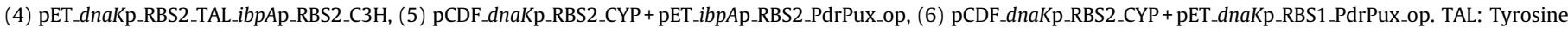
ammonia lyase; $\mathrm{C} 3 \mathrm{H}$ : 4-coumarate 3-hydroxylase; CYP: cytochrome P450 CYP199A2. Error bars are standard deviations from triplicate experiments.

during that time. Since the heat shock temperature is very high, it can cause long-term adverse effects in the production of the target compounds instead of increasing the production. In fact, the growth rate and the biomass concentration $5 \mathrm{~h}$ after the heat shock (in LB medium, before switching to M9 minimal medium) was found to be always lower than in the experiments when no heat shock was performed (Table S4). For example, thermal induction can alter the protein folding of the recombinant proteins favoring protein aggregation into inclusion bodies $[24,40]$. Slower heating rates or the overexpression of a negative feedback deficient heatshock response transcription factor $\left(\sigma^{32}\right)$ would possibly prevent this problem in the production of heterologous proteins [28,40,41]. To evaluate the effect of the heat shock or the elevated TIR value in the expression of TAL, protein gel showing the proteins present in the soluble and insoluble fractions of cell-free extracts were performed (Fig. S1 and S2). We compared the production of TAL when pCDF_dnaKp_RBS1_TAL and pCDF_dnaKp_RBS2_TAL were tested and as it can be seen the production is easily observed with the optimized RBS ( $\approx 76 \mathrm{kDa}$ ), while with the other RBS tested (RBS1) no difference is observed in protein production when compared to the empty plasmid (Fig. S1). This allowed concluding that the TIR optimization was successful. As expected, it was not possible to observe significant differences when the heat shock was performed and when the flasks were maintained at $37^{\circ} \mathrm{C}$ during the whole experiment. Also, at time zero, in pCDF_dnaKp_RBS2_TAL test, it was possible to observe the TAL production since the dnaK promoter is very active at $37^{\circ} \mathrm{C}$. Since the high TIR values may trigger the formation of inclusion bodies, a protein gel with the insoluble fraction was performed (Fig. S2). In the protein gel it can be observed a band corresponding to the TAL protein. Therefore, it is possible to conclude that a small amount of the protein may be present in inclusion bodies. However, it is also important to notice that this protein may be present due to an inefficient sonication, or due to a part of the supernatant that was maintained in the pellet when we decanted the supernatant from the falcon tube. Nonetheless, we believe that the results clearly show that there is a great benefit in optimizing TIR. In the future, a slightly lower TIR can be tested aiming to increase total protein solubility.

For the $\mathrm{C} 3 \mathrm{H}$ gene, we also tested the heat shock promoter $i b p A$ using a RBS with TIR $=86521.69$ (Fig. 3, bars (3) and (4)). The ibpA gene and other genes regulated by $i b p A$ promoter were previously found to be less expressed than the $d n a K$ at lower temperatures $\left(37^{\circ} \mathrm{C}\right)$ and highly expressed at heat shock conditions $\left(\geq 42^{\circ} \mathrm{C}\right)$. Therefore, the fold change is higher in this case than in dnaK $[33,41-44]$. Due to its characteristics, we decided to verify if this promoter could benefit the production. However, it was found that the higher fold change was not sufficient to improve caffeic acid production when compared to the experiment conducted without heat shock induction. As it can be seen in Fig. 3 (bar (3)), the caffeic acid production obtained was very low $(21.5 \mu \mathrm{M})$ and this is probably due to the usually low expression of the genes regulated by $i b p A$ promoter at $37^{\circ} \mathrm{C}$. When combined with the TAL gene (Fig. 3, bar (4), the production was higher probably due to the fact that the $p$-coumaric acid concentration is lower than when added as substrate. As mentioned above, high concentrations of this compound can be toxic to the cells and impact the production of caffeic acid. $p$-Coumaric acid production is lower when TAL is combined with $\mathrm{C} 3 \mathrm{H}$ gene since the expression of the gene in this situation proved to be lower $[18,39]$. The CYP199A2 gene was also tested with the dnaK promoter with a new RBS with higher TIR (38486.75). The expression of the redox partners was controlled by the $i b p A$ promoter using a RBS with TIR $=94670.66$. As can be seen in Fig. 3 (bar (5)), caffeic acid production using CYP199A2 and the redox partners increased 10.3 times when compared to the case where RBS1 was used (Fig. 2, bar (5)). Moreover, to evaluate if the $i b p A$ promoter was limiting the expression of Pdr and Pux, and consequently caffeic acid production, the other plasmid previously constructed with the dnaK promoter (plasmid from Fig. 2, bars (5) and (8)) was combined with the new plasmid containing CYP199A2. As seen in Fig. 3 (bar (6)), using dnaK promoter (with RBS1) to induce the expression of the redox partners increased the production 11.7 times when compared to the experiment with the same plasmid, but also with pCDF_dnaKp_RBS1_CYP(Fig. 2, bar (5)). Therefore, it can also be concluded that the experiments with different promoters (dnaK and $i b p A$ ) containing different RBS (RBS1 and 2) to express the redox partners, when compared to each other, did not exhibit statistically significant differences in the caffeic acid production (Fig. 3, bars (5) and (6)) ( $p$-value $>0.2)$. Indeed, we previously [18] concluded that the production of caffeic acid was higher when the expression of the redox partners was induced only $2.5 \mathrm{~h}$ after CYP199A2 expression. This delay in the induction allows the mitigation of the metabolic burden associated to the overexpression of several proteins simultaneously $[45,46]$. Since these redox partners are only needed to support the CYP199A2 catalytic activity when it is present, their expression can be delayed and it does not have to be too high. Therefore, the results with the $i b p A$ promoter with a RBS with very high TIR and with dnaK promoter with a RBS with medium TIR are very similar and the expression of these genes does not seem to limit caffeic acid production. 


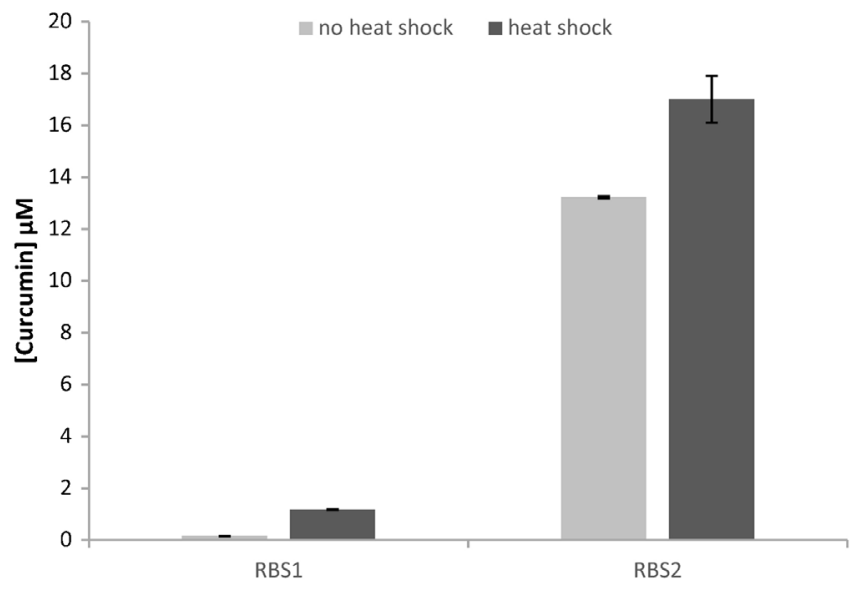

Fig. 4. Curcumin production from ferulic acid $(2000 \mu \mathrm{M})$ induced by heat using the dnaK promoter and different ribosome binding sites (RBS1 or RBS2). E. coli cells carried the three different plasmids needed to produce curcumin: pAC-4CL1, pCDF_dnaKp_RBS1/2_DCS and pRSF_dnaKp_RBS1/2_CURS1. The experiments were performed at $26^{\circ} \mathrm{C}$. Light-grey bars were maintained at this temperature through the experiment. For dark-grey bars, a heat shock was applied for $5 \mathrm{~min}$ at $48^{\circ} \mathrm{C}$.

Although the production of $p$-coumaric and caffeic acids improved by using stronger RBSs, and in $p$-coumaric acid case were even higher than when T7 promoter was used in previous studies, the production levels obtained using heat shock promoters for the caffeic acid production were still lower than those obtained using the T7 promoters. In the future, and in addition to the assessment of other RBSs, other induction times, heat shock temperatures, temperature up-shift profiles and post-induction temperatures should be tested. This can enable more or less protein expression and consequently, more or less product formation, as we reported previously [33]. In some cases, protein production at $40-42{ }^{\circ} \mathrm{C}$ [24] also proved to be very efficient. Also, oscillatory induction should be considered since it has shown to be more effective, as it leads to longer productive times that result in higher titers [28]. In addition, this strategy reduces the drawbacks associated with the use of high temperature, such as formation of inclusion bodies, acetate overproduction, decreased growth rate, plasmid instability, proteolytic susceptibility and metabolic stress caused by heterologous protein production and heat shock $[22,47]$.

\subsection{Curcumin production}

Curcumin was produced using 4-coumarate-CoA ligase (4CL1) from $A$. thaliana and diketide-CoA synthase (DCS) and curcumin synthase 1 (CURS1) from $C$. longa. This strategy was based on our previous work [17]. As in the case of caffeic acid production, the expression of curcumin genes was also triggered by heat $\left(48^{\circ} \mathrm{C}\right.$, $5 \mathrm{~min}$ ). The dnaK promoter with RBS1 was cloned in pCDFDuet-1 and pRSFDuet-1 that harbored DCS and CURS1, respectively. In the case of 4CL1, the lac promoter of pAC was not replaced since it is a constitutive promoter unlike T7 promoter, so there was no need to add expensive and toxic additives such as IPTG. In addition, the 4CL step was considered a bottleneck in curcumin production [17] given the difficulty of finding efficient 4CLs. Indeed, the 4CL1 from A. thaliana was only efficient in the pAC plasmid. Thus, the original plasmid with lac promoter was maintained.

Fig. 4 shows the production of curcumin using heat induction. The curcumin production was very low compared to the one obtained using the T7 promoter [17]. However, this was expected since the dnaK promoter is weaker than T7, and the obtained TIRs for RBS1 were not as high as desirable. In the DCS case, the TIR was 8312.53, while in the CURS1 case it was 9095.44. Although the curcumin production was low compared to the levels obtained by chemical induction, it was possible to detect it by UHPLC and a yellow color could be observed in the culture medium. Curcumin production was higher when the flasks were incubated at $26^{\circ} \mathrm{C}$ $\left(1.2 \mu \mathrm{M}-\right.$ Fig. 4 , bars (RBS1)) instead of at $37^{\circ} \mathrm{C}(0.03 \mu \mathrm{M}-$ data not shown) after the heat shock. When no heat shock was performed, only $0.15 \mu \mathrm{M}$ of curcumin was obtained at $26^{\circ} \mathrm{C}$ and no curcumin was detected by UHPLC at $37^{\circ} \mathrm{C}$. Since the dnaK promoter allows higher expression at $37^{\circ} \mathrm{C}$ than at $30^{\circ} \mathrm{C}$ [33], this temperature was first chosen as the incubation temperature instead of $26^{\circ} \mathrm{C}$. However, the production of curcumin at the higher temperature was very low (data not shown). These results suggest that the curcumin production is greatly influenced by temperature. Moreover, at $37^{\circ} \mathrm{C}$ it was found that this production was also very low when T7 promoters were used [17]. The best results obtained at $26^{\circ} \mathrm{C}$ are probably due to an improved solubility of the recombinant proteins as a consequence of a decrease of aggregation and inclusion-body formation [48].

In order to improve these results, other RBSs (RBS2) with higher TIR values were designed for DCS $(\mathrm{TIR}=42010.80)$ and CURS1 ( $\mathrm{TIR}=177340.30)$. As it is possible to observe in Fig. 4 (bars (RBS2)) the production of curcumin increased. Without heat shock the production increased 87.8 times while with the heat shock case it increased 14.4 times. Again, these results clearly highlight the importance of TIR in the design of new synthetic RBSs. In addition, in curcumin production the highest titers were achieved with the heat shock. The heat triggering probably led to an increase in gene expression of DCS and CURS1 and also of the heat shock protein genes that were beneficial for the production of curcumin.

Curcuminoids production from tyrosine or other hydroxycinnamic acids ( $p$-coumaric acid and caffeic acid) was also attempted using the pathway previously described [17]. Caffeoyl-CoA $O$ methyltransferase enzyme was used to convert caffeoyl-CoA to feruloyl-CoA. However, no production was detected using these precursors (data not shown) since the production from ferulic acid was already very low.

Although caffeic acid and curcumin production levels using heat shock promoters are still very low for industrial applications, they can be further optimized using synthetic biology approaches (e.g. synthetic scaffolds, other heat shock promoters or RBS) and other operational conditions (e.g. different temperature profiles) to reach levels similar to the ones obtained for $p$-coumaric acid that were higher than the ones obtained using T7 promoters. On the other hand, if we envisage the use of the modified bacteria producing these compounds as a result of a heat trigger for bacterial therapies (production in situ), it is important to bear in mind that the required concentrations to produce a therapeutic effect are indeed quite low. For instance, it has been proven that curcumin administration combined with ultrasound treatments highly increases the cytotoxicity and induces cellular destruction of carcinoma cells. Curcumin concentrations of 10-15 $\mu \mathrm{M}$ were shown to be very effective [49-53]. Also, in $p$-coumaric acid and caffeic acid cancer treatment studies, concentrations of $270-380 \mu \mathrm{M}$ were successfully used [1,2]. These concentrations (or even higher in some cases) can be obtained using the engineered bacteria herein developed.

In addition to the promoters that are naturally present in the E. coli HSR, other thermal regulated systems were optimized in E. coli that may be also considered to improve the yields herein reported. For example, the system composed by the lacZ operator and promoter and that is efficiently repressed by the thermossensible Lacits repressor at $30^{\circ} \mathrm{C}$, moderately active at $37^{\circ} \mathrm{C}$ [54] and totally active at $42^{\circ} \mathrm{C}$ [55] may also be a good option. Likewise, the $\lambda \mathrm{pRpL} / \mathrm{cl} 857$ system could also be tested since gene expression is inhibited at temperatures below $37^{\circ} \mathrm{C}$ and, depending on the system configuration, active at temperatures above $37^{\circ} \mathrm{C}$ (up to $42^{\circ} \mathrm{C}$ ) $[22,24]$. Although these systems are tightly regulated, basal expres- 
sion still occurs such as in the thermal regulated system studied in this work.

\section{Conclusions}

p-Coumaric acid, caffeic acid and curcumin were successfully produced in E. coli using heat shock promoters. Also, it was demonstrated that it is of utmost importance to consider the strength of the RBS when designing a biosynthetic pathway. Higher TIR values generally allow higher production. However, in metabolic engineering and synthetic biology "more is not always better." The design of RBSs with different strengths can help in the fine tuning of pathways with multiple enzymes by controlling/limiting the accumulation of intermediary by-product compounds which in turn allows maximization of the desired end-product yield. Extremely high TIR values, depending on the protein, can lead to an excess of misfolded protein. In the future, besides testing different RBSs to optimize the productions, different heat shock induction strategies should also be evaluated.

The heat-induced production of hydroxycinnamic acids and curcumin is still in its infancy and many improvements are required. However, the results gathered herein represent the first steps towards the future production of these compounds at industrial scales without chemical induction and/or possibly their use in bacterial therapies. Although titers in some cases were not very high, it is important to note that they are for example in the range required for therapeutic applications as bacterial therapies. In the future, in addition to these heat shock promoters, other promoter systems thermally regulated should be considered.

\section{Competing interests}

We declare we have no competing interests.

\section{Funding}

This study was supported by the Portuguese Foundation for Science and Technology (FCT) under the scope of the strategic funding of UID/BIO/04469/2013 unit and COMPETE 2020 (POCI-01-0145-FEDER-006684) and under the scope of the Project RECI/BBB-EBI/0179/2012 (FCOMP-01-0124-FEDER027462). The authors also acknowledge financial support from BioTecNorte operation (NORTE-01-0145-FEDER-000004) and the project MultiBiorefinery (POCI-01-0145-FEDER-016403) funded by the European Regional Development Fund under the scope of Norte2020 - Programa Operacional Regional do Norte and the Postdoctoral grant (UMINHO/BPD/37/2015) to J. L. Rodrigues funded by FCT.

\section{Appendix A. Supplementary data}

Supplementary data associated with this article can be found, in the online version, at http://dx.doi.org/10.1016/j.bej.2017.05.015.

\section{References}

[1] N.R. Prasad, A. Karthikeyan, S. Karthikeyan, B.V. Reddy, Inhibitory effect of caffeic acid on cancer cell proliferation by oxidative mechanism in human HT-1080 fibrosarcoma cell line, Mol. Cell. Biochem. 349 (2011) 11-19.

[2] S.M. Chacko, K.G. Nevin, R. Dhanyakrishnan, B.P. Kumar, Protective effect of p-coumaric acid against doxorubicin induced toxicity in H9c2 cardiomyoblast cell lines, Toxicol. Rep. 2 (2015) 1213-1221.

[3] M. Abdel-Wahab, M. El-Mahdy, M. Abd-Ellah, G. Helal, F. Khalifa, F. Hamada, Influence of p-coumaric acid on doxorubicin-induced oxidative stress in rat's heart, Pharmacol. Res. 48 (2003) 461-465

[4] K. Ikeda, K. Tsujimoto, M. Uozaki, M. Nishide, Y. Suzuki, A.H. Koyama, H. Yamasaki, Inhibition of multiplication of herpes simplex virus by caffeic acid, Int. J. Mol. Med. 28 (2011) 595-598.
[5] H. Takeda, M. Tsuji, M. Inazu, T. Egashira, T. Matsumiya, Rosmarinic acid and caffeic acid produce antidepressive-like effect in the forced swimming test in mice, Eur. J. Pharmacol. 449 (2002) 261-267.

[6] H. Utsunomiya, M. Ichinose, K. Ikeda, M. Uozaki, J. Morishita, T. Kuwahara, A.H. Koyama, H. Yamasaki, Inhibition by caffeic acid of the influenza A virus multiplication in vitro, Int. J. Mol. Med. 34 (2014) 1020-1024.

[7] R.A. Sharma, A.J. Gescher, W.P. Steward, Curcumin: the story so far, Eur. J. Cancer 41 (2005) 1955-1968.

[8] S. Prasad, S.C. Gupta, A.K. Tyagi, B.B. Aggarwal, Curcumin, a component of golden spice: from bedside to bench and back, Biotechnol. Adv. 32 (2014) 1053-1064.

[9] A. Goel, A.B. Kunnumakkara, B.B. Aggarwal, Curcumin as "Curecumin": from kitchen to clinic, Biochem. Pharmacol. 75 (2008) 787-809.

[10] A. Sarkar, R. De, A.K. Mukhopadhyay, Curcumin as a potential therapeutic candidate for Helicobacter pylori associated diseases, World J. Gastroenterol. 22 (2016) 2736.

[11] J.L. Rodrigues, K.L. Prather, L. Kluskens, L. Rodrigues, Heterologous production of curcuminoids, Microbiol. Mol. Biol. Rev. 79 (2015) 39-60.

[12] Y. Xing, H.-y. Peng, M.-x. Zhang, X. Li, W.-w. Zeng, X.-e. Yang, Caffeic acid product from the highly copper-tolerant plant Elsholtzia splendens post-phytoremediation: its extraction, purification, and identification, J. Zhejiang Univ. Sci. B 13 (2012) 487-493.

[13] S.-Y. Kang, O. Choi, J.K. Lee, B.Y. Hwang, T.-B. Uhm, Y.-S. Hong, Artificial biosynthesis of phenylpropanoic acids in a tyrosine overproducing Escherichia coli strain, Microb. Cell Fact. 11 (2012) 1-9.

[14] Y. Katsuyama, Y. Hirose, N. Funa, Y. Ohnishi, S. Horinouchi, Precursor-directed biosynthesis of curcumin analogs in Escherichia coli, Biosci. Biotechnol. Biochem. 74 (2010) 641-645.

[15] Y. Katsuyama, M. Matsuzawa, N. Funa, S. Horinouchi, Production of curcuminoids by Escherichia coli carrying an artificial biosynthesis pathway, Microbiology 154 (2008) 2620-2628.

[16] T. Furuya, Y. Arai, K. Kino, Biotechnological production of caffeic acid by bacterial cytochrome P450 CYP199A2, Appl. Environ. Microbiol. 78 (2012) 6087-6094.

[17] J. Rodrigues, R. Araújo, K. Prather, L. Kluskens, L. Rodrigues, Production of curcuminoids from tyrosine by a metabolically engineered Escherichia coli using caffeic acid as an intermediate, Biotechnol. J. 10 (2015) 599-609.

[18] J. Rodrigues, R. Araújo, K. Prather, L. Kluskens, L. Rodrigues, Heterologous production of caffeic acid from tyrosine in Escherichia coli, Enzyme Microb. Technol. 71 (2015) 36-44

[19] H. Zhang, G. Stephanopoulos, Engineering E. coli for caffeic acid biosynthesis from renewable sugars, Appl. Microbiol. Biotechnol. 97 (2013) 3333-3341.

[20] S.C. Makrides, Strategies for achieving high-level expression of genes in Escherichia coli, Microbiol. Rev. 60 (1996) 512-538.

[21] T.-Z. Su, H. Schweizer, D.L. Oxender, A novel phosphate-regulated expression vector in Escherichia coli, Gene 90 (1990) 129-133.

[22] V. Menart, S. Jevševar, M. Vilar, A. Trobiš, A. Pavko, Constitutive versus thermoinducible expression of heterologous proteins in Escherichia coli based on strong PR, PL promoters from phage lambda, Biotechnol. Bioeng. 83 (2003) 181-190.

[23] J.D. Keasling, Synthetic biology and the development of tools for metabolic engineering, Metab. Eng. 14 (2012) 189-195.

[24] N.A. Valdez-Cruz, L. Caspeta, N.O. Pérez, O.T. Ramírez, M.A. Trujillo-Roldán, Production of recombinant proteins in E. coli by the heat inducible expression system based on the phage lambda pL and/or pR promoters, Microb. Cell Fact. 9 (2010) 1-16.

[25] F. Tabandeh, S.A. Shojaosadati, A. Zomorodipour, M. Khodabandeh, M.H. Sanati, B. Yakhchali, Heat-induced production of human growth hormone by high cell density cultivation of recombinant Escherichia coli, Biotechnol. Lett. 26 (2004) 245-250.

[26] P. Srivastava, P. Bhattacharaya, G. Pandey, K. Mukherjee, Overexpression and purification of recombinant human interferon alpha2b in Escherichia coli, Protein Expr. Purif. 41 (2005) 313-322.

[27] C.A. Caulcott, M. Rhodes, Temperature-induced synthesis of recombinant proteins, Trends Biotechnol. 4 (1986) 142-146.

[28] L. Caspeta, A.R. Lara, N.O. Pérez, N. Flores, F. Bolívar, O.T. Ramírez, Enhancing thermo-induced recombinant protein production in Escherichia coli by temperature oscillations and post-induction nutrient feeding strategies, J. Biotechnol. 167 (2013) 47-55.

[29] A.M. Velez, A.C.L. Horta, A.J. da Silva, M.R.d.C. Iemma, R.d.L.C. Giordano, T.C. Zangirolami, Enhanced production of recombinant thermo-stable lipase in Escherichia coli at high induction temperature, Protein Expr. Purif. 90 (2013) 96-103.

[30] Z. Wang, Y. Wang, H. Shi, Z. Su, Expression and production of recombinant cis-epoxysuccinate hydrolase in Escherichia coli under the control of temperature-dependent promoter, J. Biotechnol. 162 (2012) 232-236.

[31] H. Wu, J. Pei, Y. Jiang, X. Song, W. Shao, pHsh vectors, a novel expression system of Escherichia coli for the large-scale production of recombinant enzymes, Biotechnol. Lett. 32 (2010) 795-801.

[32] J.C. Gupta, M. Jaisani, G. Pandey, K. Mukherjee, Enhancing recombinant protein yields in Escherichia coli using the T7 system under the control of heat inducible $\lambda P_{\mathrm{L}}$ promoter, J. Biotechnol. 68 (1999) 125-134.

[33] J.L. Rodrigues, M. Sousa, K.L. Prather, L.D. Kluskens, L.R. Rodrigues, Selection of Escherichia coli heat shock promoters towards their application as stress probes, J. Biotechnol. 188 (2014) 61-71. 
[34] D.R. Nielsen, S.H. Yoon, C.J. Yuan, K.L. Prather, Metabolic engineering of acetoin and meso-2, 3-butanediol biosynthesis in E. coli, Biotechnol. J. 5 (2010) 274-284.

[35] K.T. Watts, P.C. Lee, C. Schmidt-Dannert, Biosynthesis of plant-specific stilbene polyketides in metabolically engineered Escherichia coli, BMC Biotechnol. 6 (2006) 1-12.

[36] H.M. Salis, The ribosome binding site calculator, Methods Enzymol. 498 (2011) 19-42.

[37] S.-Y. Shin, N.S. Han, Y.-C. Park, M.-D. Kim, J.-H. Seo, Production of resveratrol from $p$-coumaric acid in recombinant Saccharomyces cerevisiae expressing 4-coumarate: coenzyme A ligase and stilbene synthase genes, Enzyme Microb. Technol. 48 (2011) 48-53.

[38] H.M. Salis, E.A. Mirsky, C.A. Voigt, Automated design of synthetic ribosome binding sites to control protein expression, Nat. Biotechnol. 27 (2009) 946-950.

[39] J.L. Rodrigues, Design and Construction of a New Biosynthetic Pathway for the Production of Curcuminoids in Escherichia Coli, University of Minho, Braga, Portugal, 2014.

[40] X. Zhang, Y. Liu, J.C. Genereux, C. Nolan, M. Singh, J.W. Kelly, Heat-shock response transcriptional program enables high-yield and high-quality recombinant protein production in Escherichia coli, ACS Chem. Biol. 9 (2014) 1945-1949.

[41] L. Caspeta, N. Flores, N.O. Pérez, F. Bolívar, O.T. Ramírez, The effect of heating rate on Escherichia coli metabolism, physiological stress, transcriptional response, and production of temperature-induced recombinant protein: a scale-down study, Biotechnol. Bioeng. 102 (2009) 468-482.

[42] K. Zhao, M. Liu, R. Burgess, The global transcriptional response of Escherichia coli to induced $\sigma^{32}$ protein involves $\sigma^{32}$ regulon activation followed by inactivation and degradation of $\sigma^{32}$ in vivo, J. Biol. Chem. 280 (2005) 17758-17768.

[43] C.S. Richmond, J.D. Glasner, R. Mau, H. Jin, F.R. Blattner, Genome-wide expression profiling in Escherichia coli K-12, Nucl. Acids Res. 27 (1999) $3821-3835$

[44] A. Rasouly, M. Schonbrun, Y. Shenhar, E.Z. Ron, YbeY, a heat shock protein involved in translation in Escherichia coli, J. Bacteriol. 191 (2009) 2649-2655.

[45] C.N.S. Santos, M. Koffas, G. Stephanopoulos, Optimization of a heterologous pathway for the production of flavonoids from glucose, Metab. Eng. 13 (2011) $392-400$.
[46] J. Wu, G. Du, J. Zhou, J. Chen, Metabolic engineering of Escherichia coli for (2S)-pinocembrin production from glucose by a modular metabolic strategy, Metab. Eng. 16 (2013) 48-55.

[47] D. Lamotte, M. Ouzzine, S. Fournel-Gigleux, J. Magdalou, J. Boudrant, A temperature profile in batch culture to increase the production of the recombinant UDP-glucuronosyltransferase 2B4 in Escherichia coli, Process Biochem. 31 (1996) 235-241.

[48] A. Correa, P. Oppezzo, Tuning different expression parameters to achieve soluble recombinant proteins in E. coli: Advantages of high-throughput screening, Biotechnol. J. 6 (2011) 715-730.

[49] X. Wang, X. Xia, A.W. Leung, J. Xiang, Y. Jiang, P. Wang, J. Xu, H. Yu, D. Bai, C. $\mathrm{Xu}$, Ultrasound induces cellular destruction of nasopharyngeal carcinoma cells in the presence of curcumin, Ultrasonics 51 (2011) 165-170.

[50] X. Wang, A.W. Leung, J. Luo, C. Xu, TEM observation of ultrasound-induced mitophagy in nasopharyngeal carcinoma cells in the presence of curcumin, Exp. Ther. Med. 3 (2012) 146-148.

[51] H.-Y. Lin, J.L. Thomas, H.-W. Chen, C.-M. Shen, W.-J. Yang, M.-H. Lee, In vitro suppression of oral squamous cell carcinoma growth by ultrasound-mediated delivery of curcumin microemulsions, Int. J. Nanomed. 7 (2012) 941-951.

[52] K.R. Carr, Y.J. Ioffe, M. Filippova, P. Duerksen-Hughes, P.J. Chan, Combined ultrasound-curcumin treatment of human cervical cancer cells, Eur. J. Obstet. Gynecol. Reprod. Biol. 193 (2015) 96-101.

[53] Y. Li, P. Wang, X. Chen, J. Hu, Y. Liu, X. Wang, Q. Liu, Activation of microbubbles by low-intensity pulsed ultrasound enhances the cytotoxicity of curcumin involving apoptosis induction and cell motility inhibition in human breast cancer MDA-MB-231 cells, Ultrason. Sonochem. 33 (2016) 26-36.

[54] Z.W. Wang, W.S. Law, Y.P. Chao, Improvement of the thermoregulated T7 expression system by using the heat-sensitive lacI, Biotechnol. Prog. 20 (2004) 1352-1358.

[55] N. Hasan, W. Szybalski, Construction of lacits and lacl q ts expression plasmids and evaluation of the thermosensitive lac repressor, Gene 163 (1995) 35-40. 